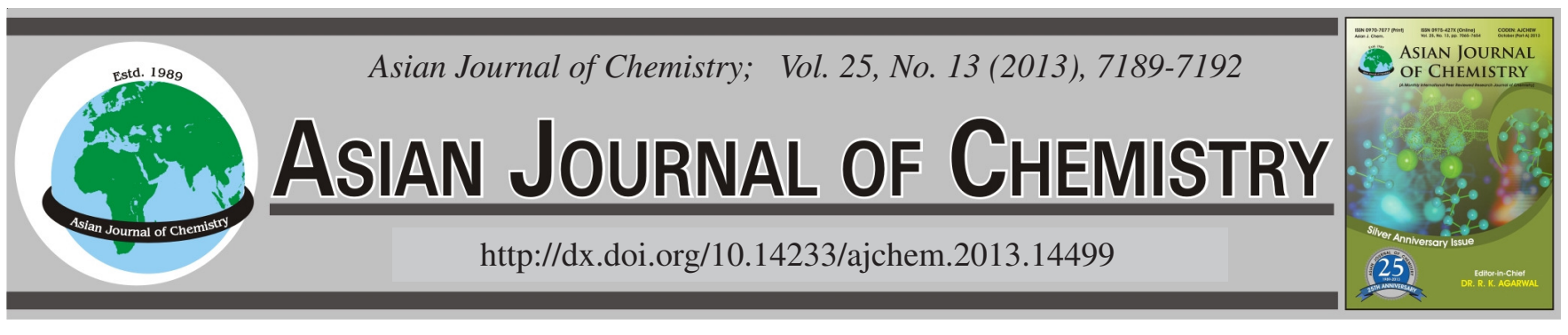

\title{
Study on the Catalytic Performance of 3-Hydroxypropionaldehyde to 1,3-Propanediol over Ni/Hydrogen Mordenite by Different Method
}

\author{
Yong Tang ${ }^{1,2, *}$, Ben-Xian Shen ${ }^{1, *}$, Chun-Li Ning ${ }^{2}$, Chun-Lei Zhang ${ }^{2}$ and Ji-Gang ZhaO ${ }^{1}$
}

${ }^{1}$ State Key Laboratory of Chemical Engineering, East China University of Science and Technology, Shanghai 200237, P.R. China

${ }^{2}$ Technology Research Institute of Shanghai Huayi Group, Shanghai, P.R. China

*Corresponding author: Fax: +86 21 64252851; Tel: +86 21 64252916; E-mail: zjg@ecust.edu.cn

(Received: 29 August 2012;

Accepted: 19 June 2013)

AJC-13670

\begin{abstract}
The catalyst for conversion of 3-hydroxypropionaldehyde to 1,3-propanediol was prepared by dry mixing, wet mixing, ion exchange and impregnation, respectively. The catalysts were characterized by transmission electron microscope and temperature programmed reduction and their catalytic performances for 3-hydroxypropionaldehyde to 1,3-propanediol were also studied. The results showed that, the carrier of hydrogen mordenite does not undermine its structure with introduction of Ni particles, the skeleton remains of its original features, the conversion of 3-hydroxypropionaldehyde was proportional with the dispersion of $\mathrm{Ni}$ on the catalyst, the stability of catalytic activity was related the binding force between the active sites of $\mathrm{Ni}$ with the carrier, the selectivity of 1,3-propanediol was more favorable with more $\mathrm{Ni}$ in the outer surface of the carrier.
\end{abstract}

Key Words: Temperature programmed reduction, 1,3-Propanediol, Catalytic performance, Ni/hydrogen mordenite.

\section{INTRODUCTION}

1,3-Propanediol (1,3-PDO) as a bifunctional organic compound could potentially be used for many synthesis reactions, in particular as a monomer for polycondensations to produce polyesters, polyethers and polyurethanes ${ }^{1}$. 1,3Propanediol may be chemically synthesized by the hydration of acrolein or by the hydroformylation of ethylene oxide to afford 3-hydroxypropionaldehyde (3-HPA). The aldehyde is hydrogenated to give 1,3-propanediol. Two other routes involve bioprocessing by certain micro-organisms ${ }^{2}$. The hydrogenation process of 3-hydroxypropionaldehyde to 1,3propanediol can be divided into two processes, the Raney nickel as the catalyst in an autoclave and supported metal catalyst in a fixed bed. The conversion of 3-hydroxypropionaldehyde and selectivity of 1,3-propanediol can reach up to $99 \%$ with the aid of Raney nickel catalyst, respectively. But the particles of Raney nickel catalyst was too small and difficult to separate products and catalysts ${ }^{3}$. So the Raney nickel catalyst for 3-hydroxypropionaldehyde to 1,3-propanediol in continuous industrial application was limited. While continuous fixed bed hydrogenation for 3-hydroxypropionaldehyde to 1,3-propanediol use the supported catalyst of the $\mathrm{Ni}$ or Pt as active components.

This paper focus on characterization and catalytic performances for 3-hydroxypropionaldehyde to 1,3-propanediol of
$\mathrm{Ni} /$ hydrogen mordenite catalyst by different method. It provide the basis for application of theory and data support for the $\mathrm{R}$ $\&$ D of efficient and stable catalyst for 3-hydroxypropionaldehyde to 1,3-propanediol.

\section{EXPERIMENTAL}

The content of 3-hydroxypropionaldehyde in water solution is $8.5 \%$, which was synthesized by acrolein hydration. $\mathrm{Ni}\left(\mathrm{NO}_{3}\right)_{2}$ is come from Sinopharm chemical reagent Co. Ltd., hydrogen mordenite (HM) of the carrier is come from fushun research institute of petroleum and petrochemicals, SINOPEC.

General procedure: The catalyst for 3-hydroxypropionaldehyde to 1,3-propanediol was prepared by dry mixing, wet mixing, ion exchange and impregnation, respectively. For the purpose of comparison, the content of $\mathrm{NiO}$ as the base in catalyst was $5 \%$.

The typical method for catalysts preparation as follows:

Dry mixing: A certain amount of $\mathrm{Ni}\left(\mathrm{NO}_{3}\right)_{2}$ and hydrogen mordenite was mixed in motar with adequate ground. Then, the mixture was placed in drying oven with the temperature of $120^{\circ} \mathrm{C}$ for overnight,remarked as A.

$\mathrm{NiO}$ was obtained by heating $\mathrm{Ni}\left(\mathrm{NO}_{3}\right)_{2}$ at $550^{\circ} \mathrm{C}$ in maffle furnace for $3 \mathrm{~h}$. Then, an amount of $\mathrm{NiO}$ and hydrogen mordenite was mixed in motar with adequate ground. Then, the mixture was placed in drying oven with the temperature of $120^{\circ} \mathrm{C}$ for overnight, remarked as B. 
Wet mixing: A certain amount of $\mathrm{Ni}\left(\mathrm{NO}_{3}\right)_{2}$, hydrogen mordenite and water was put into the motar, the mixture was worked into a paste and then with adequate ground. Then, the mixture was placed in drying oven with the temperature of $120^{\circ} \mathrm{C}$ for overnight, marked as $\mathrm{C}$.

Impregnation: A certain amount of $\mathrm{Ni}\left(\mathrm{NO}_{3}\right)_{2}$ was dissolved into water, then the carrier of hydrogen mordenite was immersed into the solution, leave it alone for $6 \mathrm{~h}$, then the solution was slowly evaporated water at water bath of $85{ }^{\circ} \mathrm{C}$, then, the mixture was placed in drying oven with the temperature of $120^{\circ} \mathrm{C}$ for overnight, marked as D.

Ion exchange: The carrier of hydrogen mordenite was immersed into a certain concentration solution of $\mathrm{Ni}\left(\mathrm{NO}_{3}\right)_{2}$, stirring for $4 \mathrm{~h}$ with water bath of $85^{\circ} \mathrm{C}$, washed, $120^{\circ} \mathrm{C}$ drying. The same operation was repeated at 4 times. The sample was marked as E.

The samples were calcined in a muffle furnace at $550{ }^{\circ} \mathrm{C}$ for $6 \mathrm{~h}, 4 \mathrm{~g}$ sample of 40-60 mesh particles was obtained by ground and screened, then the sample was reduced in the atmosphere of $\mathrm{H}_{2}$ for $4 \mathrm{~h}$ with the temperature of $400{ }^{\circ} \mathrm{C}$ and pressure of 5.0 MPa by a fixed bed reactor. Then the catalyst of Ni/hydrogen mordenite was prepared. The catalyst samples remarked as $\mathrm{Ni}-1, \mathrm{Ni}-2, \mathrm{Ni}-3, \mathrm{Ni}-4, \mathrm{Ni}-5$ were prepared samples of A, B, C, D and E, respectively.

The catalytic performance for 3-hydroxypropionaldehyde to 1,3-propanediol was carried out in a continuous-flow fixed bed reactor. The amount of catalyst loaded in the reactor was $4 \mathrm{~g}$. The typical conditions were: methanol as solvent; temperature $327 \mathrm{~K}$; pressure 5.0 MPa; the initial 3-hydroxypropionaldehyde concentration $10 \%$ (wt); the ratio of $\mathrm{H}_{2}$ to 3-hydroxypropionaldehyde was 7:1; the WHSV of feed liquid was $4.5 \mathrm{~h}^{-1}$.

Detection method: Transmission electron micrographs and selected area electron diffraction patterns were obtained using a JEOL 2011 transmission electron microscope operated at $200 \mathrm{keV}$. Samples were prepared by alcohol-drying drops of diluted solutions of the preparations supported by grids.

Temperature programmed reduction (TPR) was performed in a microflow reactor with $400 \mathrm{mg}$ of sample. After the calcinations step described above the reactor was slowly cooled to room temperature in argon flow. Noxal $\left(40 \mathrm{~mL} / \mathrm{min} 5 \% \mathrm{H}_{2}-\right.$ $95 \%$ Ar from air liquid) was then allowed to flow through the sample and $\mathrm{H}_{2}$ consumption detected with a thermal conductivity detector. The temperature was increased linearly from room temperature up to $950{ }^{\circ} \mathrm{C}$ and the rate of $10^{\circ} \mathrm{C} / \mathrm{min}$.

The feedstock and product of the reaction were analyzed on a GC-17A gas chromatography using PEG20M $(25 \mathrm{~m} \times$ $0.25 \mathrm{~mm} \times 0.5 \mu \mathrm{m}$ ) column with split mode over the traditional thermal conductively detector (TCD). The initial column temperature was $60{ }^{\circ} \mathrm{C}$ and was raised to $200{ }^{\circ} \mathrm{C}$ at a rate of $10^{\circ} \mathrm{C} /$ min and then held for $5 \mathrm{~min}$. The result of the reaction was calculated as follows:

$$
\begin{gathered}
\mathrm{X}_{\mathrm{HPA}}=\frac{\mathrm{m}_{\mathrm{HPA}}^{0}-\mathrm{m}_{\mathrm{HPA}}^{1}}{\mathrm{~m}_{\mathrm{HPA}}^{0}} \times 100 \% \\
\mathrm{Y}_{\mathrm{PDO}}=\frac{74 \times \mathrm{m}_{\mathrm{PDO}}}{76 \times \mathrm{m}_{\mathrm{HPA}}^{0}} \times 100 \%
\end{gathered}
$$

$$
\mathrm{S}_{\mathrm{PDO}}=\frac{\mathrm{Y}_{\mathrm{PDO}}}{\mathrm{X}_{\mathrm{HPA}}} \times 100 \%
$$

$\mathrm{X}_{\mathrm{HPA}}, \mathrm{Y}_{\mathrm{PDO}}$ and $\mathrm{S}_{\mathrm{PDO}}$ denote the conversion of 3-hydroxypropionaldehyde (HPA), the yields of 1,3-propanediol ( $\left.\mathrm{Y}_{\mathrm{PDO}}\right)$ and the selectivity of 1,3-propanediol ( $\left.\mathrm{S}_{\mathrm{PDO}}\right)$, respectively. The $\mathrm{m}_{\mathrm{HPA}}^{1}$ and $\mathrm{m}_{\mathrm{PDO}}$ stand for the mass content of hydroxypropionaldehyde and 1,3-propanediol, respectively. The $\mathrm{m}_{\mathrm{HPA}}^{0}$ was the initial mass content of hydroxypropionaldehyde, respectively.

\section{RESULTS AND DISCUSSION}

TEM picture of Ni/hydrogen mordenite catalysts by different method was shown in Fig. 1. It can be seen that, for sample of Ni-1by dry mixing, the Ni particles in carrier was almost at the outer surface of each particle with uniform size distribution and the Ni particle size was larger. For sample of $\mathrm{Ni}-2$ by dry mixing, the diameter of Ni particle was larger, but small than the one in $\mathrm{Ni}-1$, that was to say, the dispersion was better than $\mathrm{Ni}-1$. For sample of Ni-3 by wet mixing and Ni-4 by impregnation, the dispersion of $\mathrm{Ni}$ particles was significantly improved and particle size decreased significantly. For sample of Ni-5 by ion exchange, the Ni particle was not observed,
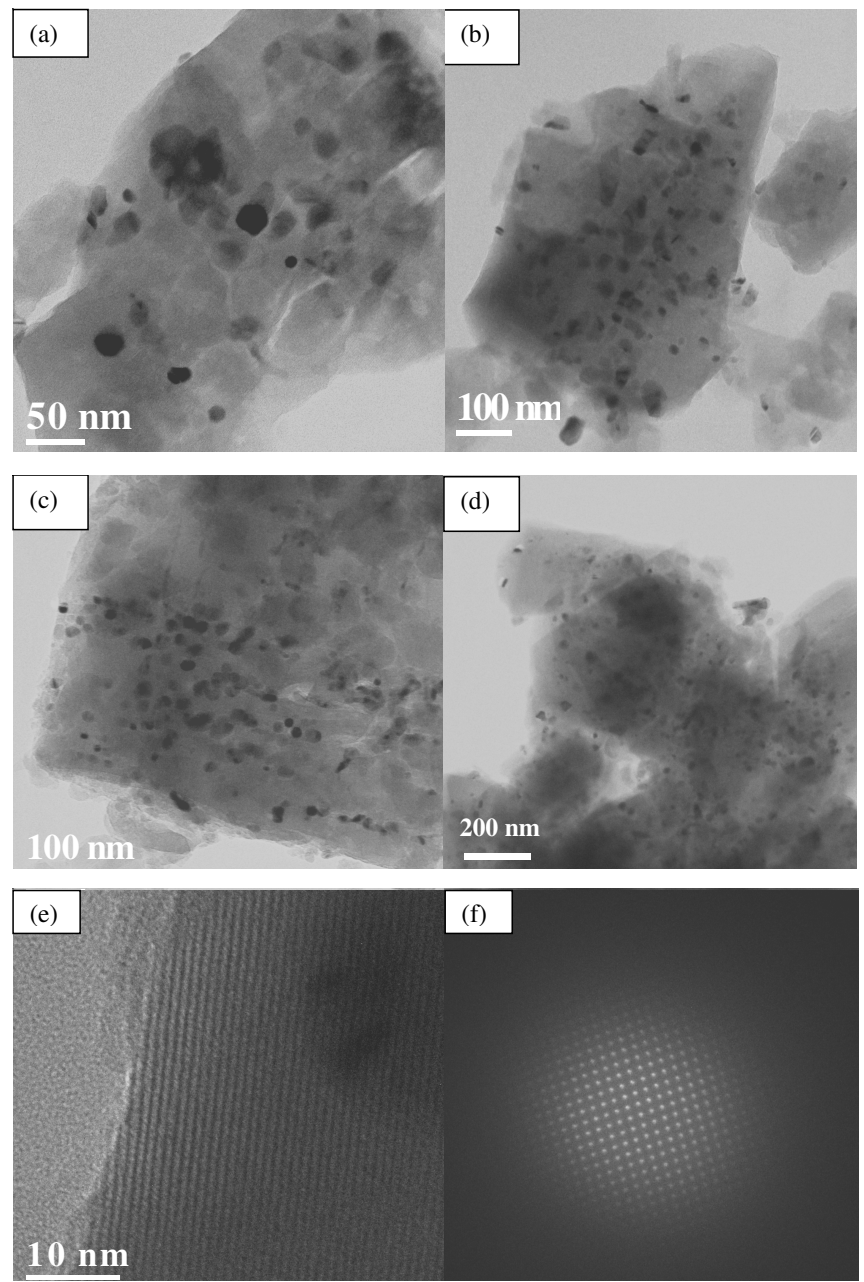

Fig. 1. TEM picture of Ni/hydrogen mordenite catalysts by different method. (a) Ni-1; (b) Ni-2; (c) Ni-3; (d) Ni-4; (e) Ni-5; (f) SAED of Ni-3 
this was for the Ni particles inside the channels with exchange sites in the carrier and on the other hand, the Ni particles may be smaller particles and highly dispersion and the amount was limited.

Fig. 1(f) shows the SAED picture of $\mathrm{Ni}-3$ by wet mixing. It can be seen that the structure of the sample was integrity and the SAED pictures of other samples was the same as the one of Ni-3. It illustrated that the carrier of hydrogen mordenite does not undermine its structure with introduction of $\mathrm{Ni}$ particles, the skeleton remains of its original features.

Temperature programmed reduction (TPR): Temperature programmed reduction spectrum of $\mathrm{Ni} /$ hydrogen mordenite catalysts by different method was shown in Fig. 2. It can be seen that the TPR patterns of Ni-1 and Ni-5 were significantly different from the other samples. For the Ni-1 catalyst, there was only a large reduction peak with low temperature of $356{ }^{\circ} \mathrm{C}$, combination with the above TEM characterization, the peak should be attributed to the accumulation of large particles $\mathrm{NiO}$ reduction peak ${ }^{4}$. For the weak intermolecular forces between the big particles of $\mathrm{NiO}$ with the carrier, so it was reduced more easily. In other words, the reduction temperature was low. For the Ni-5 catalyst, there was only a reduction peak with high temperature of $651^{\circ} \mathrm{C}$, the corresponding exchange sites in the zeolite on the $\mathrm{Ni}$ species. Because this part of $\mathrm{Ni}$ and strong interaction between carriers and their zeolite played a stabilizing role, it is difficult to be reduced, so the reduction temperature is higher. For $\mathrm{Ni}-2, \mathrm{Ni}-3$ and Ni-4 catalysts, there are two reduction peaks, in addition to low temperature reduction peak of $360^{\circ} \mathrm{C}$, also a high temperature reduction peak of $560{ }^{\circ} \mathrm{C}$. It means that the surface of these three catalysts have two different states of $\mathrm{NiO}$. That means low temperature reduction peak corresponding to the reunion of the large particles of NiO. High temperature reduction peak, corresponding to the highly dispersed, small particle size $\mathrm{NiO}, \mathrm{NiO}$ are expected to enter this part of the pores of the zeolite, in the highly dispersed. And because of smaller particle size, so the above TEM characterization was not detected in the presence of these particles.

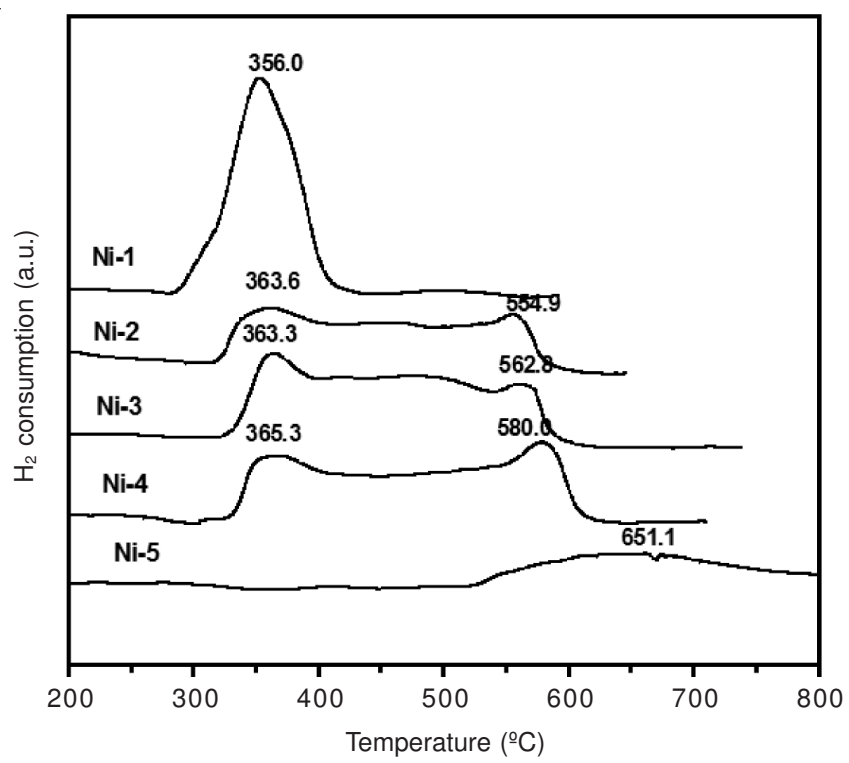

Fig. 2. TPR spectrum of Ni/hydrogen mordenite catalysts by different method
In the TPR experiments, the sample peak area presented the amount of hydrogen consumption in the reduction process, which was related with the amount of oxygen species in the samples. It can be seen that the high temperature reduction peak area of Ni-4 was significantly larger than the other two, it indicated that good dispersion of Ni-4 sample, may be more small particles into the channels of zeolite. The high temperature reduction peak of $\mathrm{Ni}-2$ and $\mathrm{Ni}-3$ was roughly equal size.

Catalytic experiments: Effect of $\mathrm{Ni} /$ hydrogen mordenite catalysts by different method on $\mathrm{X}_{\mathrm{HPA}}$ for hydroxypropionaldehyde to 1,3-propanediol was shown in Fig. 3. The $\mathrm{X}_{\mathrm{HPA}}$ order for hydroxypropionaldehyde to 1,3-propanediol with the reaction time of $2 \mathrm{~h}$ was as follows: $\mathrm{Ni}-4>\mathrm{Ni}-3>\mathrm{Ni}-2>$ Ni-1 $>$ Ni-5. From the characterization by the TEM and TPR, the $\mathrm{X}_{\mathrm{HPA}}$ was proportional with the dispersion of $\mathrm{Ni}$ on the catalyst. For the theory of several Ni catalyst loading the same terms as the base to $\mathrm{NiO}$ were $5 \%$ (wt), Ni dispersion of particles larger exposed surface of the catalyst the greater the number of active sites and the $\mathrm{X}_{\mathrm{HPA}}$ was high. The catalytic performance of Ni-5 was very poor for the limited number of active sites and the exchange bit of $\mathrm{NiO}$ was difficult to be reduced to active site of $\mathrm{Ni}$.

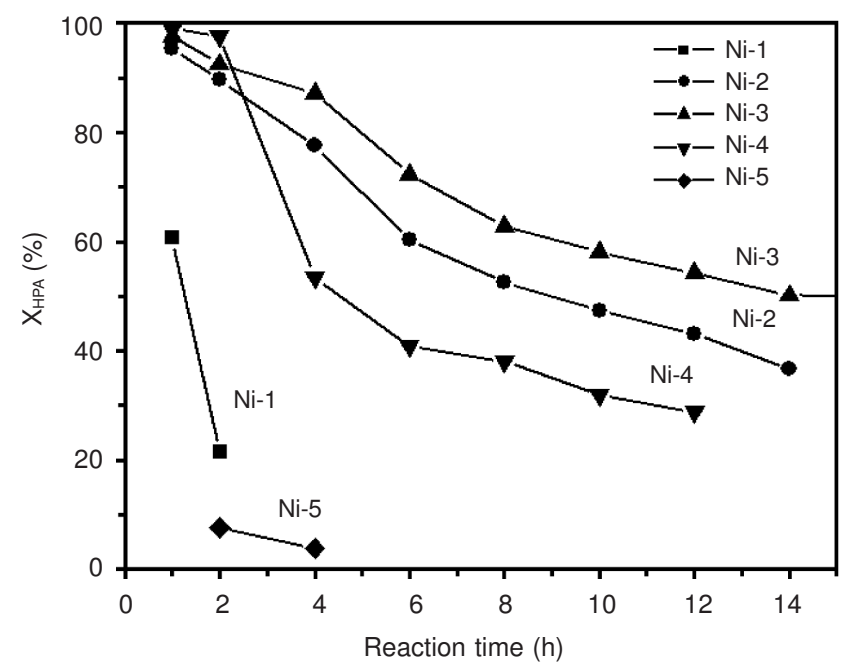

Fig. 3. Effect of Ni/hydrogen mordenite catalysts by different method on $\mathrm{X}_{\mathrm{HPA}}$ for hydroxypropionaldehyde to 1,3-propanediol

The catalyst deactivation of Ni-1 was very fast, the $\mathrm{X}_{\mathrm{HPA}}$ has declined almost $40 \%$ in the first reaction time of $1 \mathrm{~h}$. The $\mathrm{Ni}$ particles in $\mathrm{Ni}-1$ was relative larger than the other one and easily reunite with each other, for the force between $\mathrm{Ni}$ species with the support was weak (It can be seen from TPR characterization). The Ni was easy loss during the reaction, which may be caused by a sharp decline for $\mathrm{Ni}-1$ in its main activity. For the impregnation of Ni-4 catalyst activity also decreased rapidly $4 \mathrm{~h}$ response, the activity of $\mathrm{X}_{\mathrm{HPA}}$ decreased from 98.9 to $53.5 \%$. Probably because more Ni particles dispersed within the carrier channel, this part of the Ni particles in the reaction process because of the loss of catalytic activity of pore blockage. The $\mathrm{X}_{\mathrm{HPA}}$ of Ni-2 and Ni-3 catalyst is relatively stable.

Effect of $\mathrm{Ni} /$ hydrogen mordenite catalysts by different method on $\mathrm{S}_{\mathrm{PDO}}$ for hydroxypropionaldehyde to 1,3-propanediol was shown in Fig. 4. The $\mathrm{S}_{\mathrm{PDO}}$ order for hydroxypropionaldehyde to 1,3-propanediol was as follows: Ni-1 $>\mathrm{Ni}-2>\mathrm{Ni}-3>\mathrm{Ni}-4$ 


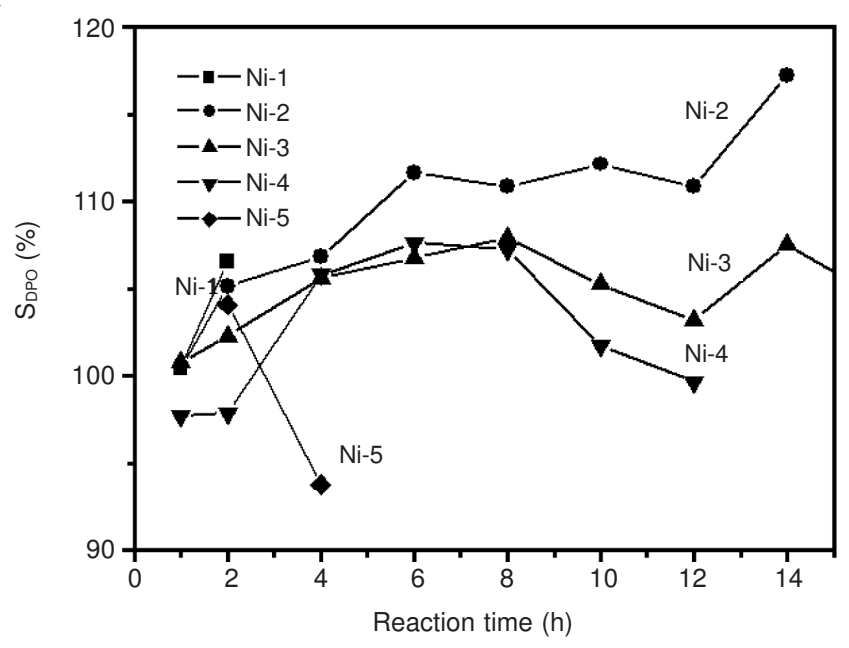

Fig. 4. Effect of Ni/hydrogen mordenite catalysts by different method on $\mathrm{S}_{\mathrm{DPO}}$ for hydroxypropionaldehyde to 1,3-propanediol

$>$ Ni-5. That means the selectivity of 1,3-propanediol was more favorable with more $\mathrm{Ni}$ in the outer surface of the carrier.

\section{Conclusion}

The catalyst for 3-hydroxypropionaldehyde to 1,3propanediol was prepared by dry mixing, wet mixing, ion exchange and impregnation, respectively. The catalysts were characterized by TEM and TPR and their catalytic performances for 3-hydroxypropionaldehyde to 1,3-propanediol were also studied. The results showed that, the carrier of hydrogen mordenite does not undermine its structure with introduction of Ni particles, the skeleton remains of its original features, the catalyst activity of $\mathrm{X}_{\mathrm{HPA}}$ was proportional with the dispersion of $\mathrm{Ni}$ on the catalyst, the stability of catalytic activity was related the binding force between the active sites of $\mathrm{Ni}$ with the carrier, the selectivity of 1,3-propanediol was more favourable with more $\mathrm{Ni}$ in the outer surface of the carrier. It provide the basis for application of theory and data support for the R \& D of efficient and stable catalyst for 3-hydroxypropionaldehyde to 1,3-propanediol.

\section{REFERENCES}

1. Y. Tang, B.X. Shen, C.L. Ning, C.L. Zhang and Y.P. Jie, Acta Petrolei Sin., 26, 538 (2010).

2. R.K. Saxena, Pinki, S. Saran and J. Isar, Biotechnol. Adv., 27, 895 (2009).

3. S.C. Chen, C.C. Chu, F.S. Lin, J.Y. Chou and C.C. Huang, Modified Raney Nickel Catalyst and a Process for Preparing Diols by Using the same, US Patent 5888923 (1996).

4. Z.H. Xu, S.Z. Guo and C.R. Gu, Chin. J. Catal., 25, 897 (2004). 